\title{
Lipidomic analysis of polyunsaturated fatty acids and their oxygenated metabolites in plasma by solid-phase extraction followed by LC-MS.
}

\author{
Authors \\ Gabriel Dasilva ${ }^{\mathrm{a}^{*}}$, Manuel Pazos ${ }^{\mathrm{a}}$, José M. Gallardo ${ }^{\mathrm{a}}$, Isaac Rodríguez ${ }^{\mathrm{b}}$, Rafael Cela ${ }^{\mathrm{b}}$, Isabel Medina ${ }^{\mathrm{a}}$. \\ ${ }^{a}$ Instituto de Investigaciones Marinas, Consejo Superior de Investigaciones Científicas (IIM-CSIC), E- \\ 36208 Vigo, Galicia, Spain \\ ${ }^{b}$ Department of Analytical Chemistry, Nutrition and Bromatology and Research Institute for Food \\ Analysis (I.I.A.A.), University of Santiago de Compostela, E-15782 Santiago de Compostela, Galicia, \\ Spain \\ *Corresponding author. Tel. +34986231930; fax: +34986292762. E-mail address: \\ gabrieldasilva@iim.csic.es (Gabriel Dasilva)
}

\begin{abstract}
The present work describes the development of a robust and sensitive targeted analysis platform for the simultaneous quantification in blood plasma of lipid oxygenated mediators and fatty acids using Solid-phase Extraction (SPE) and high performance liquid chromatography coupled to tandem mass spectrometry (HPLC-MS/MS). The concurrent analysis of these lipid mediators is challenging because of their instability, differences in solubility and the often occurrence of isobaric forms with similar fragmentation patterns. Results demonstrated that the reduction of SPE temperature to $4^{\circ} \mathrm{C}$ is a critical parameter for preserving the hydroperoxy derivatives. Polymeric HLB cartridges increased 40-50\% ARA, EPA and DHA sensitivity compared to C18 sorbent, and also provided higher global performance for most hydroxides and other oxidation products. The proposed method yields for two tested mass analyzers high sensitivity, good linearity and reproducibility, with detection limits ranging $0.002-7 \mathrm{ng} / \mathrm{mL}$ and global recoveries as high as $85-112 \%$. However, it should be noted the additional advantage of the linear ion trap (LIT) mass analyzer acquiring in full scan product ion mode, compared to the triple quadrupole (QqQ) acquiring in Multiple Reaction Monitoring (MRM): the full scan product ion mode provides the full fragmentation spectra of compounds that allowed to discriminate coeluting isomers and false positive identifications without additional chromatography development. The proposed lipidomic procedure demonstrates to be confident, simple and sensitive to profile in plasma a wide range of lipid eicosanoid and docosanoid mediators, including innovatively the analysis of hydroperoxy congeners and non-oxidized PUFA precursors.
\end{abstract}

\section{Keywords}

Oxygenated lipid mediators, solid-phase extraction, liquid chromatography, mass spectrometry, triple quadrupole, ion trap.

\section{Introduction}

Polyunsaturated fatty acids (PUFAs) exhibit a range of biological effects, many of which are mediated by the formation of lipid derivatives. Such metabolites are produced in vivo through the action of cyclooxygenases (COXs), lipooxygenases (LOXs), cytochrome P450 monooxygenases (CYP450) and/or free radical oxidation mechanisms [1]. PUFAs with twenty carbons like arachidonic acid (ARA) and eicosapentaenoic acid (EPA) are precursors of many eicosanoids like prostanoids (prostaglandins and tromboxanes) and leukotrienes, a family of compounds involved in inflammatory processes [2]. From ARA, series-2 prostanoids like $\mathrm{PGE}_{2}, \mathrm{PGD}_{2}$ or $\mathrm{TXB}_{2}$ and series-4 leukotrienes like $\mathrm{LTB}_{4}$ and also hydroxyeicosatetraenoic acids (HETEs) are generated by COXs and LOXs activity [3]; whilst CYP450 and autoxidation reactions result in various hydroxy-, hydroperoxy-, epoxy-fatty acids and $\mathrm{F}_{2}$-isoprostanes [4]. In a similar 
manner, from EPA derive many lipid compounds including series-3 prostanoids (e.g., $\mathrm{PGE}_{3}, \mathrm{PGD}_{3}$ and $\mathrm{TXB}_{3}$ ), series-5 leukotrienes (e.g., LTB ${ }_{5}$ ), hydroxy- and hydroperoxyeicosapentaenoic acids (HEPEs and HpEPEs) [5][6]. From EPA also derive $\mathrm{F}_{3}$ isoprostanes (a group of analgesic compounds) [2][7], and series-E resolvins (e.g., $\mathrm{RvE}_{1}$ and $\mathrm{RvE}_{2}$ ) which are a novel discovered family of trihydroxy congeners that result from COX-2 activity and act as anti-inflammatory mediators [3][8].

Enzymatic oxidation or autoxidation of docosahexaenoic acid (DHA) produces hydroxy- docosahexaenoic acids (HDoHEs) and hydroperoxy- docosahexaenoic acids (HpDoHEs); moreover, it has been recently shown that DHA gives rise to a novel family of strong anti-inflammatory compounds termed neuroprostanes with dihydroxy (protectins, PD) and trihydroxy (series-D resolvins) structures [3][8]. (Figure S1 of Electronic Supplementary Material shows the similar formation cascades of ARA, EPA and DHA fatty acids).

Oxygenated metabolites of PUFAs have an important role in a wide range of biological functions. They are part of membrane cells, activate gene transcription, and are also involved in many diseases and inflammatory processes [9][10]. Therefore, these lipid mediators have been investigated in biomarkers discovery and drug development studies [3]. Specific examples include the pro-inflammatory role of ARA eicosanoids and isoprostanes metabolites in many processes related to oxidative stress, such as hypercholesterolemia [11], liver cirrhosis [12], Crest syndrome [13], myocardial reperfusion [14] or type-2 diabetes [15][16]. On the other hand, many hydroxy derived compounds of EPA and DHA are involved in the regulation of vascular tone [17], arteriosclerosis [18] or Alzheimer [19], and are considered markers of lipid peroxidation. Finally, novel studies have related anti-inflammatory and cellular protective activities of $\mathrm{RvE}_{1}, \mathrm{RvD}_{1}$ and $\mathrm{PD}_{1}$ [20] to benefits in cardiovascular diseases, dry eyes or even Alzheimer [21][22].

The analytical determination of oxygenated PUFA metabolites is a challenging task, mainly because of their low physiological levels and large number of isomers with very similar physicochemical properties [10]. Therefore, a sensitive and selective detection procedure following an effective separation step is essential to comprehensively study this class of lipids [23]. Methods used for the determination of these lipid mediators currently involve an extraction and/or pre-concentration step followed by the chromatographic separation and mass spectrometry determination. The most common Solid-phase extraction (SPE) sorbents are C18 conventional cartridges [16][24][25], and polymeric sorbents like Oasis-HLB [26][27][28] or Strata-X [29][30]. For many years, gas chromatography coupled to mass spectrometry (GC/MS) was the method of choice for eicosanoids analysis [15][17][31]. Nowadays, the widespread liquid chromatography/mass spectrometry (LC-MS) coupled with high-sensitivity electrospray ionization (ESI) has provided a new approach for quantification, minimizing sample preparation requirements and particularly avoiding derivatization reactions [3][24][25][32][33]. The triple quadrupole (QqQ) mass spectrometer working in the multiple reaction monitoring (MRM) mode [16][26][28][29][30], together with hybrid variants such as quadrupole ion trap (Q-Trap) [27][34] and quadrupole time-of-flight (Q-TOF) [35][36], have become the MS technology of choice for analysis of oxygenated PUFA metabolites. 
The aim of this research was to develop an analytical methodology based on SPE and LC-MS, to allow the simultaneous detection and quantification of a wide range of lipid mediators in plasma. PUFA derivatives like prostaglandins, leukotrienes, tromboxanes, hydroxy and hydroperoxy acids, isoprostanes, resolvins and protectins derived from ARA, EPA and DHA and their fatty acid precursors were the target analytes. The proposed method innovatively attempts to analyze PUFA metabolites with a wide range of polarity (octanol-water partition coefficients ranged from 1.1 of $\mathrm{PGD}_{3}$ to 7.3 of DHA), concentration (up to 4 orders of magnitude), stability diversity (hydroperoxides are highly unstable), and applies polymeric HLB cartridges for the extraction of hydroxy and hydroperoxy PUFA congeners. Two different mass spectrometers were tested in order to discuss and compare both acquisition modes in terms of false positive identifications: a QqQ acquiring in the MRM mode that is commonly applied to lipidomic approaches, and a Linear Ion Trap (LIT) acquiring in full scan product ion mode. Their analytical parameters for optimal sensitivity and selectivity were addressed. Applicability of the analytical platform was validated by analyzing plasma samples of genetically obese spontaneously hypertensive rats.

\section{Experimental}

\subsection{Standards, solvents and sorbents}

Thromboxane $\mathrm{B}_{3}\left(\mathrm{TXB}_{3}, 9 \alpha-( \pm) 11,15(\mathrm{~S})\right.$-trihydroxy-thromba-5Z,13E,17Z-trien-1-oic acid), prostaglandin $\mathrm{D}_{3}\left(\mathrm{PGD}_{3}, 9 \alpha-15(\mathrm{~S})\right.$-dihydroxy-11-oxo-prosta-5Z,13E, 17Z-trien-1oic acid), prostaglandin $\mathrm{E}_{3}\left(\mathrm{PGE}_{3}, 11 \alpha-15\right.$ (S)-dihydroxy-9-oxo-prosta-5Z,13E,17Ztrien-1-oic acid), prostaglandin $\mathrm{E}_{2}$ ( $\mathrm{PGE}_{2}, 11 \alpha-15(\mathrm{~S})$-dihydroxy-9-oxo-prosta-5Z,13Edien-1-oic acid), resolvin $\quad \mathrm{D}_{1} \quad\left(\mathrm{RvD}_{1}, \quad 7(\mathrm{~S}), 8(\mathrm{R}), 17(\mathrm{~S})\right.$-trihydroxy4Z,9E,11E,13Z,15E,19Z-docosahexaenoic acid), protectin $\mathrm{D}_{1} \quad \mathrm{PD}_{1}, 10(\mathrm{~S}), 17(\mathrm{~S})-$ dihydroxy-4Z,7Z,11E,13Z,15E,19Z-docosahexaenoic acid), leukotriene $\mathrm{B}_{4}$ (LTB 5(S),12(R)-dihydroxy-6Z,8E,10E,14Z-eicosatetraenoic acid), isoprostane $\mathrm{F}_{2 \alpha}$ (8isoPGF $2 \alpha, 9 \alpha, 11 \alpha, 15(\mathrm{~S})$-trihydroxy- $8 \beta$-prosta-5Z,13E-dien-1-oic acid), isoprostane $\mathrm{F}_{3 \alpha} \quad$ (8isoPGF $3 \alpha, \quad 9 \alpha, 11 \alpha, 15(\mathrm{~S})$-trihydroxy-8$\beta$-prosta-5Z,13E,17Z-trien-1-oic acid), 15HpEPE (15(S)-hydroperoxy-5Z,8Z,11Z,13E,17Z-eicosapentaenoic acid), 12HpEPE (12(S)-hydroperoxy-5Z,8Z,10E,14Z,17Z-eicosapentaenoic acid), 17HpDoHE (17(S)hydroperoxy-4Z,7Z,10Z,13Z,15E,19Z-docosahexaenoic acid), 15HEPE ( \pm 15 -hydroxy5Z,8Z,11Z,13E,17Z-eicosapentaenoic acid), 12HEPE ( \pm 12 -hydroxy5Z,8Z,10E,14Z,17Z-eicosapentaenoic acid), 5HEPE ( \pm 5-hydroxy-6E,8Z,11Z,14Z,17Zeicosapentaenoic acid), 11HETE ( \pm 11 -hydroxy-5Z,8Z,12E,14Z-eicosatetraenoic acid), 12HETE-d $_{8} \quad$ (12(S)-hydroxy-5Z,8Z,10E,14Z-eicosatetraenoic-5,6,8,9,11,12,14,15-d 8 acid), 17HDoHE ( \pm 17-hydroxy-4Z,7Z,10Z,13Z,15E,19Z-docosahexaenoic acid), 11HDoHE ( \pm 11-hydroxy-4Z,7Z,9E,13Z,16Z,19Z-docosahexaenoic acid), 4HDoHE ( \pm 4-hydroxy-5E,7Z,10Z,13Z,16Z,19Z-docosahexaenoic acid), eicosapentaenoic acid (EPA, 5Z,8Z,11Z,14Z,17Z-eicosapentaenoic acid), docosahexaenoic acid (DHA, 4Z,7Z,10Z,13Z,16Z,19Z-docosahexaenoic acid) and arachidonic acid (ARA, 5Z,8Z,11Z,14Z-eicosatetraenoic acid) were purchased from Cayman Chemicals (Ann Arbor, MI, USA). The suppliers stated purities higher than $96 \%$ for all standards.

Stock standard solutions of all analytes were prepared in ethanol and stored at $-80{ }^{\circ} \mathrm{C}$ under nitrogen. Individual stocks $(5 \mu \mathrm{g} / \mathrm{mL})$ were used to optimize ESI ionization and MS/MS fragmentation conditions by flow injection analysis.

For most species, calibration standard solutions were prepared in the range between 1 and $500 \mathrm{ng} / \mathrm{mL}$. Two additional calibration solutions (1000 and $1500 \mathrm{ng} / \mathrm{mL})$ were 
made for 12HEPE. A second series of calibration standards from 100 to $50000 \mathrm{ng} / \mathrm{mL}$ was prepared for parent PUFAs (EPA, ARA, DHA) due to their higher concentration in plasma samples. The internal standard $\left(11\right.$ HETE- $\left.\mathrm{d}_{8}\right)$ was included in all calibration solutions at a constant level of $500 \mathrm{ng} / \mathrm{mL}$. Calibration lines were calculated by the least-squares linear regression method with the internal standard correction.

Methanol, Optima LC-MS grade, was purchased from Fisher Scientific (New Jersey, USA); ultrapure water was obtained from a Milli-Q system (Millipore, Bedford, MA, USA); methyl formate was purchased from Sigma Aldrich (Poole, UK); n-hexane was provided by Merk (Darmstadt, Germany) and ethanol, formic acid and hydrochloric acid were from AnalR Normapur (Fontenai, France). SPE cartridges (C18-SepPak, 100 $\mathrm{mg}, 1 \mathrm{~mL}$ and Oasis-HLB, $60 \mathrm{mg}, 3 \mathrm{~mL}$ ) were supplied by Waters (Milford, MA, USA).

\subsection{Samples and sample preparation}

Plasma samples were collected from female SHROB (genetically obese spontaneously hypertensive) rats, an animal model of metabolic syndrome. Animals were kept in an isolated room with a constantly regulated temperature $\left(22 \pm 2{ }^{\circ} \mathrm{C}\right)$ and humidity $(50 \pm 10 \%)$ in a $12 \mathrm{~h}$ artificial light cycle, and fed ad libitum with water and a standard pelleted chow diet A04 from Harlan Ibérica (Barcelona, Spain), which contained on wet basis $60 \%$ carbohydrate, $16 \%$ protein and $3 \%$ fat. Rats were sacrificed by exsanguination, blood was collected in tubes with ethylenediaminetetraacetic acid (EDTA) to obtain plasma (as described below). All the procedures performed agreed with the national and institutional guidelines of the Animal Care and Use Committee at the CSIC.

Blood was centrifuged at $850 \mathrm{~g}\left(4^{\circ} \mathrm{C}, 15 \mathrm{~min}\right)$ in the presence of EDTA to remove erythrocytes. Then, plasma was supplemented with $5 \mathrm{mM}$ phenylmethylsulfonyl fluoride (PMSF, protease inhibitor) and erythrocyte free samples were immediately stored at $-80{ }^{\circ} \mathrm{C}$ until use. A pre-treatment step for small fluids was applied to plasma samples before the SPE [37]. The defrosting process was carried out slowly in darkness, to prevent possible analytes oxidation and degradation. Samples $(90 \mu \mathrm{L})$ were diluted with cold methanol $30 \%(\mathrm{v} / \mathrm{v})$ to a final volume of $1 \mathrm{~mL}$, and spiked with the internal standard (IS) 11HETE- $\mathrm{d}_{8}$. Samples were incubated on ice for $10 \mathrm{~min}$ and then centrifuged at $5000 \mathrm{rpm}$ for $10 \mathrm{~min}$, at $4{ }^{\circ} \mathrm{C}$, to remove potential proteins that may cause interference. Supernatant and washes of the resultant pellet with $30 \%$ methanol were collected in amber glass vials and added to SPE cartridges.

SPE extractions were carried out in a cold room at $4{ }^{\circ} \mathrm{C}$. Cartridges were conditioned with $5 \mathrm{~mL}$ methanol followed by $5 \mathrm{~mL}$ Milli-Q water. After sample loading, cartridges were sequentially washed with $5 \mathrm{~mL} 15 \%$ methanol (v/v), $5 \mathrm{~mL}$ Milli-Q water and 2.5 $\mathrm{mL}$ hexane. Then, the sorbent phase was dried under a stream of nitrogen for $30 \mathrm{~min}$ and analytes were eluted using $2 \mathrm{~mL}$ methyl formate. The extraction procedure was performed using a vacuum manifold; the vacuum was adjusted so that individual drops could be seen from each cartridge. Extracts were evaporated to dryness under a fine stream of nitrogen; the residue was dissolved in $30 \mu \mathrm{L}$ ethanol and stored at $-80{ }^{\circ} \mathrm{C}$ prior to LC-MS/MS analysis.

It is important to notice that during the sample spiking procedure, precursor fatty acids could be degradated rending hydroperoxy derivates. Also, impurities existing in the 
commercial standards of EPA, ARA and DHA can become important since their addition level was huge in relation to the rest of compounds. To avoid these potential problems, three sets of spiked samples were prepared in order to separately evaluate the recoveries of precursor fatty acids, hydroperoxy and the rest of analytes. Addition levels used in these sets of spiked samples were $150 \mathrm{ng} / \mathrm{mL}$ for PUFAs metabolites, except for 12HEPE $(600 \mathrm{ng} / \mathrm{mL})$. Their precursors were added to plasma at much higher levels: EPA $(3 \mu \mathrm{g} / \mathrm{mL})$, DHA $(15 \mu \mathrm{g} / \mathrm{mL})$ and ARA $(45 \mu \mathrm{g} / \mathrm{mL})$, which are in the same order of concentration as existing in non-spiked (blank) samples. The added concentration of IS $\left(12 \mathrm{HETE}_{\mathrm{d}} \mathrm{d}_{8}\right.$ ) was $500 \mathrm{ng} / \mathrm{mL}$ in all experiments.

Different recovery experiments were performed according to the step procedure where the compounds were spiked: at the beginning of the process (Global recovery, SPE-LCESI-MS/MS), after SPE (Matrix effect, ESI-MS/MS) or comparing initial spiked samples with additions done after SPE (SPE recovery). The repeatability of the methodology was expressed as percentage of relative standard deviation (\%RSD).

\subsection{Apparatus}

Analyses were carried out on two LC-MS systems. One system consists of two ProStar 210 high-pressure mixing pumps (Varian, Walnut Creek, CA, USA), a Metachem Technologies vacuum membrane degasser (Bath, UK), and an autosampler and thermostated column compartment ProStar 410 module (Varian). This LC is coupled with a triple quadrupole 320-MS equipped with an electrospray interface (Varian). Nitrogen, used as nebulising and drying gas, is provided by a nitrogen generator (Domnick Hunter, Durham, UK). Argon (99.999\%) was used as collision gas. Instrument control and data acquisition were done by Varian MS Workstation software.

The other LC system consists of an Agilent 1260 Series (Agilent, Palo Alto, CA) that includes a binary pump, a degasser system and a thermostated autosampler, coupled to a linear ion trap (LIT) mass spectrometer LTQ Velos Pro equipped with an electrospray interface (Thermo Fisher, Rockford, IL, USA). Nitrogen was used as nebulising and drying gas and helium was the collision gas. Instrument control and data acquisition were done with Xcalibur software.

A Waters C18-Symmetry column, $150 \times 2.1 \mathrm{~mm}, 3.5 \mu \mathrm{m}$ (Milford, MA, USA) protected with a $4 \times 2 \mathrm{~mm}$ C18 guard cartridge provided by Phenomenex (Torrance, CA, USA) was used to perform LC separations in both systems. SPE clean-up and extraction steps were performed using a vacuum manifold system from Supelco (Bellefonte, PA, USA) coupled to a vacuum pump from Millipore (Bedford, MA, USA). Plasma samples were foremost centrifuged in an Avanti J25 refrigerated centrifuge (Beckman Coulter, USA).

\subsection{LC-MS/MS conditions}

A binary eluent system of water (A) and methanol (B), both with $0.02 \%$ (v/v) of formic acid, was used as mobile phase in the LC separation. The gradient was: 0-1 min $(60 \%$ B), 2-12 $\min (80 \% \mathrm{~B}), 13-23 \mathrm{~min}(100 \% \mathrm{~B})$, and $25-30 \mathrm{~min}(60 \% \mathrm{~B})$. The flow rate was set to $0.2 \mathrm{~mL} / \mathrm{min}$, the column effluent was directly introduced in the ESI interface without splitting, and injection volume was set to $10 \mu \mathrm{L}$. The column was maintained at room temperature and extracts were kept at $-20{ }^{\circ} \mathrm{C}$ right up the injection moment. Retention times for target compounds are shown in Table 1.

\subsection{1. $Q q Q$ system}


The ESI interface was operated in the negative mode with a needle potential of $-4 \mathrm{kV}$, a source temperature of $50{ }^{\circ} \mathrm{C}$, a desolvation temperature of $200{ }^{\circ} \mathrm{C}$, a nebulising gas pressure of $55 \mathrm{psi}(380 \mathrm{kPa})$ and a drying gas pressure of $20 \mathrm{psi}(138 \mathrm{kPa})$. Argon pressure in the collision cell was kept at 2 mTorr for MS/MS measurements. The mass window of the first and last quadrupoles was adjusted to 2.0 and $1.5 \mathrm{amu}$, respectively.

Selection of the most intense MS/MS transitions was done by direct infusion of individual standard solutions $(5 \mu \mathrm{g} / \mathrm{mL})$ at a flow rate of $20 \mu \mathrm{L} / \mathrm{min}$. The intensity of the $[\mathrm{M}-\mathrm{H}]^{-}$ion was optimized by varying the capillary voltage, and subsequently, the [Mproduct ions were obtained by varying the collision energy ( 0 to $60 \mathrm{eV})$ with the aid of the automated routines included in the software package. For each analyte, the two most intense or selective transitions were selected (Table 1).

\subsubsection{LIT system}

Operating conditions of the ESI source were negative ion mode with a sheath gas flow rate of 40 units, spray voltage of $5.5 \mathrm{kV}$, capillary temperature of $300{ }^{\circ} \mathrm{C}$ and $\mathrm{S}$-lens radio-frequency level of $60 \%$. Mass spectrometer was tuned optimizing voltages on the lenses and trap conditions whilst infusing standard solutions in the same way as QqQ system. The collision induced dissociation (CID) energy was optimized for each compound in order to maximize the intensity of their product ions. The identification of targeted compounds was helped with the full ion product spectra recorded in the range from 90 to $400 \mathrm{~m} / \mathrm{z}$ units. To quantify the analytes, the most intense and selective transition was used. Individual MS/MS parameters for each compound are summarized in Table 1.

\section{Results and discussion}

\subsection{Optimization of LC-ESI-MS/MS conditions}

Table 1 summarizes the optimal MS/MS transitions selected for the QqQ and LIT assays. These transitions were recorded in order to facilitate the individual identification of the compounds minimizing further false positive identifications in biological samples. Once transitions were set, compounds were chromatographically separated on a C18 column using a gradient of water/methanol solvents with $0.02 \%$ formic acid $(\mathrm{v} / \mathrm{v})$. Several compounds coeluted under the same chromatographic peak, but they could be individually quantified using specific transitions except for the particular case of the coeluting isomers $\mathrm{PGE}_{3}$ and $\mathrm{PGD}_{3}$ that also showed the same fragmentation pattern (Figure S2 of Electronic Supplementary Materialpresents their individual mass spectrum). $\mathrm{MS}^{3}$ fragmentations tested for both prostaglandins in the LIT spectrometer did not provide further qualitative information; therefore, they were quantified together.

The LC separation cycle takes $30 \mathrm{~min}$, including a 5 min column conditioning step before the next injection. Overall, this running time was comparable with other reported LC methods of eicosanoids analysis and provides the basis for a rapid assay [4][38]. Some of the selected product ions for QqQ were in agreement with the available literature values as the following: $\mathrm{TXB}_{3} 367>169$, 8iso- $\mathrm{PGF}_{2 \alpha} 353>192, \mathrm{PGE}_{2}$ 351>271, 5HEPE $317>115$ or 17 HDoHE $343>281$. However, for these compounds, the found LIT transitions were different than those previously reported. Transitions associated to $\mathrm{RvD}_{1}, \mathrm{LTB}_{4}, 12 \mathrm{HEPE}$ or 11HETE matched with literature values described for both equipment [3][24][28][29]. Figure 1 shows representative chromatograms of the analysis of these compounds. 


\subsection{Detection method validation}

Table 2 shows the values of linearity (from 1 to $500 \mathrm{ng} / \mathrm{mL}$ for oxidized derivates and 100 to $50000 \mathrm{ng} / \mathrm{mL}$ for PUFAs), instrumental LODs and LOQs (defined for a signal to noise ratio of 3 and 10, respectively), and repeatability estimated by either of the two mass detectors. The standard calibration functions confirmed that both equipment achieved linear responses for all compounds, $\mathrm{R}^{2}$ ranged between 0.9860 and 0.9999 . Acceptable precision was achieved by both instruments; RDSs \% were lower than $12 \%$ except for hydroperoxy compounds at $5 \mathrm{ng} / \mathrm{mL}$ level.

Finally, instrumental LODs and LOQs for the QqQ system were respectively estimated to be in the range of $0.003-7$ and $0.01-23 \mathrm{ng} / \mathrm{mL}$, and that is in agreement with those previously reported in literature [3][16][24][26][28][30][39]. LIT mass spectrometer achieved respectively LODs and LOQs between 0.002-3 and 0.006-10 ng/mL, values similar to those reported in a hybrid system consisting on a quadrupole-ion trap mass analyzer (QTrap) operating in MRM mode [27]. No previous reports were found using a linear ion trap spectrometer operating in full scan product ion mode.

\subsection{Optimization of solid-phase extraction (SPE)}

3.3.1 Breakthrough and elution solvent volume, washing conditions and extraction sorbent.

In preliminary SPE experiments, aqueous samples (simulating plasma conditions) were used to optimize the SPE process trying two different cartridges, $60 \mathrm{mg}$ Oasis-HLB and $100 \mathrm{mg} \mathrm{C} 18$. Methyl formate and ethanol were tested as eluting solvents achieving the same performance; even though, methyl formate was selected due to its higher volatility that facilitates the later stage of concentration. Three consecutive $2 \mathrm{~mL}$ fractions of organic solvent were collected from SPE cartridges, previously spiked with $50 \mu \mathrm{L}$ of a mixture solution of target compounds $(200 \mathrm{ng} / \mathrm{mL})$. Responses measured in the first fraction represented more than $98 \%$ of the total for all compounds. Thus, $2 \mathrm{~mL}$ of methyl formate were the optimal eluting conditions employed in further experiments. This extract was concentrated to dryness and finally re-constituted with $30 \mu \mathrm{L}$ of ethanol.

The breakthrough volume of both sorbents was investigated using up to $3 \mathrm{~mL}$ spiked aqueous samples passed through two cartridges connected in series $(\mathrm{C} 18>\mathrm{C} 18$ and HLB>HLB) and eluted separately. This sample volume, $3 \mathrm{~mL}$, did not achieve the sorbent breakthrough volume due to none of the compounds were detected in the extract from the second cartridge, neither C18 nor HLB.

Considering biological and complex samples, many authors have demonstrated the importance of a washing sample step during the SPE procedure to reduce sample complexity and to prevent matrix effects during LC-ESI-MS determination. Washing conditions were set to $5 \mathrm{~mL}$ of water followed by $5 \mathrm{~mL}$ of $15 \%$ methanol and $2.5 \mathrm{~mL}$ of hexane [16][24][26][30] after verifying that none of the compounds were lost in the washing fraction.

Recoveries attained with Oasis-HLB and C18 cartridges were compared for $2 \mathrm{~mL}$ of spiked water samples at a final concentration of $150 \mathrm{ng} / \mathrm{mL}$ per compound. The comparative recovery results showed that HLB cartridges performed better than C18 ones for precursor fatty acids and hydroxy compounds, for which HLB recoveries 
ranged from 70 to $98 \%$ while those were reduced to 42 to $82 \%$ with the C18 sorbent (Figure 2). Eicosanoids, resolvins and protectins were eluted with similar recoveries (70 to $105 \%$ ) in both sorbents (Figure 2), and they are in agreement with other published results using HLB [24][25] and C18 [26][27]. Hydroperoxy compounds recoveries were higher using HLB sorbent than $\mathrm{C} 18$, but they still ranged from 50 to $70 \%$, so that further optimization of the process was required. On the basis of results depicted in Figure 2, it was decided to select the HLB sorbent (never tested for hydroxy and hydroperoxy metabolites) to continue with optimization of the extraction process.

\subsubsection{Effect of sample pH, organic modifiers addition and temperature.}

When the SPE conditions described in the above section for aqueous solutions were applied to plasma samples, a decrease in the yield of SPE process was noticed for all compounds. Thus, additional variables were evaluated in order to better understand the behavior of the analytes during the sample preparation process and, obviously, to improve the yield of sample preparation.

Some authors have suggested that acidification of plasma to $\mathrm{pH} 3$ before extraction, improves the affinity of fatty acids and their derivatives by reversed-phase sorbents, and thus the efficiency of SPE extraction [3][40][41]. However, we found similar efficiencies for acidified ( $\mathrm{pH} 3$ ) and non-acidified plasma samples (data not shown); thus, the $\mathrm{pH}$ of plasma was not modified.

The three fatty acids involved in this study (ARA, EPA and DHA) and their hydroxy and hydroperoxy derivatives are relatively lipophilic compounds; thus, these compounds are more prone to be lost by lipophilic interactions with the pellet formed after sample centrifugation, and that could partially explain their low recoveries obtained. In order to verify this hypothesis, a series of experiments were performed by diluting spiked plasma samples $(90 \mu \mathrm{L})$ with aqueous solutions $(1 \mathrm{~mL})$ containing different percentages of methanol from 0 to $50 \%$. After centrifugation, the supernatants were submitted to the SPE procedure and absolute recoveries calculated (Figure 3). In general, recoveries obtained for most compounds increased with the percentage of added methanol from 0 to $30 \%$ (quantitative recoveries were achieved with $30 \%$ of methanol). The exception to this trend corresponded to the free fatty acids (ARA, EPA and DHA). Quantitative recoveries for these species were only achieved using a 50\% of methanol, which is in agreement with their high octanol-water partition coefficients $\left(\mathrm{K}_{\mathrm{ow}}\left(\mathrm{ARA}, 25^{\circ} \mathrm{C}\right)=6.99, \mathrm{~K}_{\mathrm{ow}}\left(\mathrm{EPA}, 25^{\circ} \mathrm{C}\right)=6.50, \mathrm{~K}_{\mathrm{ow}}\left(\mathrm{DHA}, 25^{\circ} \mathrm{C}\right)=7.26\right)$. However, methanol proportions above $30 \%$, reduced the recovery for the more water-soluble species (eicosanoids, resolvins and protectins) due to breakthrough problems in the cartridge. Thus, 30\% of methanol was the optimized organic modifier used to favor the extraction of PUFAs minor metabolites in plasma. In the specific case of hydroperoxy compounds, the best recoveries were also obtained with $30 \%$ of methanol.

The influence of the temperature during the extraction process was then checked comparing spiked plasma processed at room temperature and $4{ }^{\circ} \mathrm{C}$. Our results demonstrated that temperature had a critical impact on hydroperoxy compounds; whilst it did not affect the rest of studied analytes (Figure 4). Hydroperoxy recoveries were around $50 \%$ at room temperature, whereas such recoveries increased to $90-100 \%$ at 4 ${ }^{\circ} \mathrm{C}$. Therefore, this is a critical parameter to be controlled in the global extraction process of lipid mediators. 
To summarize, the optimal extraction conditions were set as follows: $90 \mu \mathrm{L}$ of internal standard-spiked plasma were diluted with $1 \mathrm{~mL} 30 \%$ methanol. After centrifugation, the supernatant was recovered and loaded into the barrel of an Oasis-HLB 60mg SPE cartridge. The entire process was performed at $4{ }^{\circ} \mathrm{C}$. A scheme of optimized SPE method is provided as Electronic Supplementary Material in Figure S3.

\subsection{Repeatability, SPE-LC-MS recoveries and matrix effects}

Table 3 summarizes the repeatability of the entire optimized method, the overall recoveries for the SPE and SPE-LC-MS procedures, and the matrix effects for spiked plasma samples to a final concentration of $150 \mathrm{ng} / \mathrm{mL}$ per compound except for 12HEPE $(600 \mathrm{ng} / \mathrm{mL})$, EPA $(3 \mu \mathrm{g} / \mathrm{mL})$, DHA $(15 \mu \mathrm{g} / \mathrm{mL})$ and ARA $(45 \mu \mathrm{g} / \mathrm{mL})$, using 60 mg Oasis-HLB cartridges.

Good and similar repeatabilities were obtained for the compounds using the two analyzers, with the exception of hydroperoxy analytes. Repeatabilities were ranged between RSD values of 1-15\% (13-26\% for hydroperoxy) by SPE-LC-ESI-QqQ and 3$13 \%$ (11-25\% for hydroperoxy) by SPE-LC-ESI-LIT system. Thus, the precision of the method is controlled by the sample preparation step rather than by the characteristics of the mass analyzer.

Global recoveries for LIT ranged from 80 to $112 \%$, and were generally higher than QqQ global recoveries. In particular, the QqQ system provided global recoveries as low as a $27-38 \%$ for the three hydropexides (15HpEPE, 12HpEPE and 17HpDoHE) and the hydroxyl 4HDoHE. Global recoveries for RvD1 and PUFA precursors (ARA, EPA and DHA) were also significantly lower, 50-71\%, with the QqQ configuration. SPE recoveries were around $100 \%$ in both equipments with the exception of $\mathrm{RvD}_{1}(67-75 \%)$. Thus, according to the results compiled in Table 3, the main parameter that affected the global recovery of analytes was the matrix effect.

It is known that the sensitivity and accuracy of the mass spectrometry determinations can be influenced by the coelution of matrix components with ability to reduce or enhance the intensity of $[\mathrm{M}-\mathrm{H}]^{-}$precursor ions. Matrix effects observed for the QqQ were higher than for the LIT. Seven compounds, i.e. the three hydroperoxides, 4HDoHE and the three PUFA precursors, showed suppression of $28-71 \%$ of their signal with the QqQ, whilst with the LIT system only the precursor DHA and 11HDoHE exhibit matrix effect by losing their signal up to $29-20 \%$, respectively. There might be multiple reasons for the different matrix effects observed on the two systems. Ion suppression mainly occurs in the early stages of the ionization process; therefore, the difference in matrix effect contribution may be in part due to the particular design of the ESI source of each system [42].

\subsection{Application to plasma analysis}

The optimized SPE-LC-MS/MS method was applied to plasma samples of genetically obese spontaneously hypertensive rats. Samples were analyzed by both instrumental systems and results were compared. As it has been assessed above, both equipments achieved similar quantitative performance in the analysis of standard compounds; however, the MRM acquisition mode (QqQ) gave several false identifications when it was applied to rat plasma samples. The QqQ system was not able to distinguish targeted compounds from other coeluting isomers with similar MRM transitions. It should be noted that oxygenated PUFA metabolites comprise an elevated number of isomeric and 
isobaric species that may coelute [23]. In contrast, the full ion product mode (LIT) provided full MS/MS spectra which help to minimize the number of false positive identifications. As example, when plasma samples were analyzed by MRM mode, 11HDoHE was tentatively identified based on 343>121 $\mathrm{m} / \mathrm{z}$ quantification and 343>149 $\mathrm{m} / \mathrm{z}$ identification transitions; and on the retention time 18.33 and $18.25 \mathrm{~min}$ for the sample and standard solution (Figure 5a,b). However, the LIT MS/MS spectra obtained for the same peak revealed that its fragmentation pattern is different from the 11HDoHE standard (Figure 5c,d). This peak was probably a different coeluting hydroxy derivate of DHA, based on the typical fragments attributed to neutral loses of water (343>325 and $299>281 \mathrm{~m} / \mathrm{z})$ and $\mathrm{CO}_{2}(343>299)$ that were detected in both CID spectra. Different fragment ions from C-C cleavages were found (343>233, 255, 161 in the sample and $343>194,165,149$ in the standard). There are at least other 14 bioactive lipids with the same exact mass than 11HDoHE (MW: 344.235) [23], that in the case of coelution may explain the observed false positive identification in plasma analysis. In a similar way, the full ion product mode confirmed the false positive identification of 15HEPE, 5HEPE and 4HDoHE using the MRM mode.

Quantitative results obtained with the LIT instrument are presented in Table 4. Eight of twenty-three searched compounds were identified. ARA was the metabolite found at highest level (59446 ng/mL) and $\mathrm{TXB}_{3}$ was that present at the lowest concentration (3 $\mathrm{ng} / \mathrm{mL}$ ). Due to the concentration level of $17 \mathrm{HpDoHE}$ was higher than the maximum level of calibration, it was necessary to dilute the samples.

\section{Conclusions}

The present work describes the development of a robust and sensitive targeted analysis platform for the simultaneous quantification in blood plasma of a larger number of eicosanoids/docosanoids (tromboxanes, prostaglandins, resolvins, protectins, leukotrienes, isoprostanes, hydroxy and hydroperoxy) which are quantified jointly to their PUFA precursors (ARA, EPA and DHA). The developed method overcame issues associated to the wide range of polarity (octanol-water partition coefficients ranged from 1.1 of $\mathrm{PGD}_{3}$ to 7.3 of DHA), concentration (up to 4 orders of magnitude) and stability diversity (hydroperoxides are highly unstable) of these metabolites. Two mass detectors were tested: the most common used QqQ instrument operating in MRM and LIT spectrometer acquiring in full ion product scan mode. Although the QqQ assays were highly sensitive and selective and chromatographic separation was optimized, the full ion product scan acquisition on the LIT analyzer was more appropriated to minimize false positive identifications for these metabolites. The optimized methodology is simple, sensitive, high-throughput and lends itself to lipidomic applications. The method can be used to biological applications targeted for biomarker discovery or other therapeutic and pharmacological approaches. It can be combined with information on metabolic, proteomic and genomic profiles providing global evidence for evaluating the role of lipids at cellular, tissue and system level.

\section{Acknowledgements}

This work was supported by the Spanish Ministry of Science and Innovation (Grants AGL2009-12374-C03-01, -02, and -03). The Consejo Superior de Investigaciones Científicas (CSIC) and the University of Santiago de Compostela (USC) are gratefully acknowledged for the doctoral fellowship to Gabriel Dasilva. Xunta de Galicia and European Social Fund are also thankfully recognized by the financial support of the postdoctoral "Isidro Parga Pondal" contract to Manuel Pazos. 


\section{References}

1. Frankel EN (1998) Lipid Oxidation. The Oily Press LTD, West Ferry, Dundee, Scotland

2. Mathews CK, Van Holde KE, Ahern KG (2003) In: Capella I (ed) Biochemistry, $3^{\text {a }}$ edn. Adison Wesley, Oregon

3. Masoodi M, Mir AA, Petasis NA, Serhan CN, Nicolaou A (2008) Simultaneous lipidomic analysis of three families of bioactive lipid mediators leukotrienes, resolvins, protectins and related hydroxy-fatty acids by liquid chromatography/electrospray ionisation tandem mass spectrometry. Rapid Communications in Mass Spectrometry. Doi: $10.1002 / \mathrm{rcm} .3331$

4. Nithipatikom K, DiCamelli R, Kohler S, Gumina R, Falck J, Campbell W, Gross G (2001) Determination of cytochrome P450 metabolites of arachidonic acid in coronary venous plasma during ischemia and reperfusion in dogs. Anal Biochem. Doi:10.1006/abio.2001.5044

5. Yin H, Brooks JD, Gao L, Porter NA, Morrow JD (2007) Identification of novel autoxidation products of the omega-3 fatty acid eicosapentaenoic acid in vitro and in vivo. J Biol Chem. Doi:10.1074/jbc.M703108200

6. Kamal-Eldin A, Yanishlieva N (2002) N-3 fatty acids for human nutrition: stability considerations. European Journal of Lipid Science and Technology. Doi:10.1002/14389312(200212)104:12<825::AID-EJLT825>3.0.CO;2-N

7. Gao L, Yin H, Milne G, Porter N, Morrow J (2006) Formation of F-ring isoprostanelike compounds (F-3-isoprostanes) in vivo from eicosapentaenoic acid. J Biol Chem. Doi:10.1074/jbc.M601035200

8. Massey KA, Nicolaou A (2011) Lipidomics of polyunsaturated-fatty-acid-derived oxygenated metabolites. Biochem Soc Trans. Doi:10.1042/BST0391240

9. Sun Y, Oh SF, Uddin J, Yang R, Gotlinger K, Campbell E, Colgan SP, Petasis NA, Serhan CN (2007) Resolvin D1 and its aspirin-triggered 17R epimer - Stereochemical assignments, anti-inflammatory properties, and enzymatic inactivation. J Biol Chem. Doi:10.1074/jbc.M609212200

10. Min L, Li Y, Yu B, Huwei L (2014) Analytical Methods in Lipidomics and their Applications. Analytical Chemistry 86 (1):161

11. Davi G, Alessandrini P, Mezzetti A, Minotti G, Bucciarelli T, Costantini F, Cipollone F, Bon G, Ciabattoni G, Patrono C (1997) In vivo formation of 8-epiprostaglandin F-2 alpha is increased in hypercholesterolemia. Arteriosclerosis Thrombosis and Vascular Biology

12. Pratico D, Iuliano L, Basili S, Ferro D, Camastra C, Cordova C, FitzGerald G, Violi F (1998) Enhanced lipid peroxidation in hepatic cirrhosis. J Invest Med 
13. Stein C, Tanner S, Awad J, Roberts L, Morrow J (1996) Evidence of free radicalmediated injury (isoprostane overproduction) in scleroderma. Arthritis Rheum. Doi:10.1002/art.1780390711

14. Delanty N, Reilly M, Pratico D, Lawson J, McCarthy J, Wood A, Ohnishi S, 5 Fitzgerald D, FitzGerald G (1997) 8-Epi PGF(2 alpha) generation during coronary

6 reperfusion - A potential quantitative marker of oxidant stress in vivo. Circulation Plasma 8-Epi-Pgf(2-Alpha) Levels are Elevated in Individuals with Non-InsulinDependent Diabetes-Mellitus. FEBS Lett. Doi:10.1016/0014-5793(95)00649-T

16. Cavalca V, Minardi F, Scurati S, Guidugli F, Squellerio I, Veglia F, Dainese L, Guarino A, Tremoli E, Caruso D (2010) Simultaneous quantification of 8-isoprostaglandin-F-2 alpha and 11-dehydro thromboxane B-2 in human urine by liquid chromatography-tandem mass spectrometry. Anal Biochem. Doi:10.1016/j.ab.2009.10.014

17. Walter M, Blumberg J, Dolnikowski G, Handelman G (2000) Streamlined F-2isoprostane analysis in plasma and urine with high-performance liquid chromatography and gas chromatography/mass spectroscopy.

Anal Biochem. Doi:10.1006/abio.1999.4476

18. Waddington E, Sienuarine K, Puddey I, Croft K (2001) Identification and quantitation of unique fatty acid oxidation products in human atherosclerotic plaque using high-performance liquid chromatography. Anal Biochem. Doi:10.1006/abio.2001.5075 KA, Nicolaou A, Lynch MA (2011) The polyunsaturated fatty acids, EPA and DPA exert a protective effect in the hippocampus of the aged rat. Neurobiol Aging. Doi:10.1016/j.neurobiolaging.2010.04.001

20. Serhan CN, Gotlinger K, Hong S, Lu Y, Siegelman J, Baer T, Yang R, Colgan SP, Petasis NA (2006) Anti-inflammatory actions of neuroprotectin D1/protectin D1 and its natural stereoisomers: Assignments of dihydroxy-containing docosatrienes. Journal of 30 Immunology

21. Arita M, Bianchini F, Aliberti J, Sher A, Chiang N, Hong S, Yang R, Petasis NA, Serhan CN (2005) Stereochemical assignment, antiinflammatory properties, and 33 receptor for the omega-3 lipid mediator resolvin E1. J Exp Med. 34 Doi:10.1084/jem.20042031 Lipidomics Analysis of Bioactive Lipids in Complex Regulatory Networks. Anal Chem. Doi:10.1021/ac1015563 
24. Masoodi M, Nicolaou A (2006) Lipidomic analysis of twenty-seven prostanoids and isoprostanes by liquid chromatography/electrospray tandem mass spectrometry. Rapid Communications in Mass Spectrometry. Doi:10.1002/rcm.2697

25. Kretschmer A, Giera M, Wijtmans M, de Vries L, Lingeman H, Irth H, Niessen WMA (2011) Derivatization of carboxylic acids with 4-APEBA for detection by positive-ion LC-ESI-MS(/MS) applied for the analysis of prostanoids and NSAID in urine. Journal of Chromatography B. Doi:10.1016/j.jchromb.2010.11.028

26. Zhang H, Il'yasova D, Sztaray J, Young SP, Wang F, Millington DS (2010) Quantification of the oxidative damage biomarker 2,3-dinor-8-isoprostaglandin-F-2 alpha in human urine using liquid chromatography-tandem mass spectrometry. Anal 11 Biochem. Doi:10.1016/j.ab.2009.12.024

27. Yang J, Schmelzer K, Georgi K, Hammock BD (2009) Quantitative Profiling Method for Oxylipin Metabolome by Liquid Chromatography Electrospray Ionization Sanz JM, Gil-Izquierdo A (2012) A ultra-pressure liquid chromatography/triple quadrupole tandem mass spectrometry method for the analysis of 13 eicosanoids in human urine and quantitative 24 hour values in healthy volunteers in a controlled constant diet. Rapid Communications in Mass Spectrometry. Doi:10.1002/rcm.6224

29. Deems R, Buczynski MW, Bowers-Gentry R, Harkewicz R, Dennis EA (2007) Detection and quantitation of eicosanoids via high performance liquid chromatographyelectrospray ionization-mass spectrometry. Lipidomics and Bioactive Lipids: MassSpectrometry-Based Lipid Analysis. Doi:10.1016/S0076-6879(07)32003-X

30. Taylor AW, Bruno RS, Traber MG (2008) Women and smokers have elevated urinary $\mathrm{F}(2)$-isoprostane metabolites: A novel extraction and LC-MS methodology. Lipids. Doi:10.1007/s11745-008-3222-1

31. Nourooz-Zadeh J (2008) Key issues in F-2-isoprostane analysis. Biochem Soc

32. Levandi T, Pussa T, Vaher M, Toomik P, Kaljurand M (2009) Oxidation products of free polyunsaturated fatty acids in wheat varieties. European Journal of Lipid Science and Technology. Doi:10.1002/ejlt.200800286

33. Maskrey BH, O'Donnell VB (2008) Analysis of eicosanoids and related lipid mediators using mass spectrometry. Biochem Soc Trans. Doi:10.1042/BST0361055

34. Kortz L, Geyer R, Ludwig U, Planert M, Bruegel M, Leichtle A, Fiedler GM, Thiery J, Ceglarek U (2009) Simultaneous eicosanoid profiling and identification by liquid chromatography and hybrid triple quadrupole-linear ion trap mass spectrometry for metabolomic studies in human plasma. Laboratoriumsmedizin-Journal of Laboratory Medicine. Doi:10.1515/JLM.2009.057 
1 35. Dickinson J, Murphy R (2002) Mass spectrometric analysis of leukotriene A(4) and 2 other chemically reactive metabolites of arachidonic acid. J Am Soc Mass Spectrom. 3 Doi:10.1016/S1044-0305(02)00456-7

4 36. Li M, Feng B, Liang Y, Zhang W, Bai Y, Tang W, Wang T, Liu H (2013) Lipid 5 profiling of human plasma from peritoneal dialysis patients using an improved $2 \mathrm{D}$ 6 (NP/RP) LC-QToF MS method. Analytical and Bioanalytical Chemistry. 7 Doi:10.1007/s00216-013-7109-5

8 37. Massey KA, Nicolaou A Lipidomics of oxidized polyunsaturated fatty acids. Free $9 \quad$ Radical Biology and Medicine. Doi:10.1016/j.freeradbiomed.2012.08.565

10 38. Margalit A, Duffin K, Isakson P (1996) Rapid quantitation of a large scope of 11 eicosanoids in two models of inflammation: Development of an electrospray and 12 tandem mass spectrometry method and application to biological studies. Anal Biochem. 13 Doi:10.1006/abio.1996.0093

14 39. Ferreiro-Vera C, Maria Mata-Granados J, Priego-Capote F, Manuel Quesada15 Gomez J, Dolores Luque de Castro M (2011) Automated targeting analysis of 16 eicosanoid inflammation biomarkers in human serum and in the exometabolome of stem 17 cells by SPE-LC-MS/MS. Analytical and Bioanalytical Chemistry.

18 Doi:10.1007/s00216-010-4400-6

19 40. Durn JH, Marshall KM, Farrar D, O'Donovan P, Scally AJ, Woodward DF, 20 Nicolaou A (2010) Lipidomic analysis reveals prostanoid profiles in human term 21 pregnant myometrium. Prostaglandins Leukotrienes Essential Fatty Acids. 22 Doi:10.1016/j.plefa.2009.11.002

23 41. McDaniel JC, Massey K, Nicolaou A (2011) Fish oil supplementation alters levels 24 of lipid mediators of inflammation in microenvironment of acute human wounds. 25 Wound Repair and Regeneration. Doi:10.1111/j.1524-475X.2010.00659.x

26 42. Jessome L, Volmer D (2006) Ion suppression: A major concern in mass 27 spectrometry. Lc Gc North America 


\section{Captions}

\section{Figure captions}

4

5 Figure 1: Representative LC-MS/MS chromatograms of the 23 lipid mediators (75

$6 \mathrm{ng} / \mathrm{mL}$, per compound) provided by the LIT instrument.

8 Figure 2: Comparative SPE recovery results between $60 \mathrm{mg}$ HLB and $100 \mathrm{mg}$ C18

9 cartridges, of $2 \mathrm{~mL}$ spiked water samples with a final concentration of $150 \mathrm{ng} / \mathrm{mL}$ per compound. The same extraction conditions were applied, at room temperature and neutral $\mathrm{pH}, \mathrm{n}=3$ replicates.

Figure 3: Comparative recovery results for the extraction with $60 \mathrm{mg}$ HLB cartridges of 14 spiked plasma samples (final concentration of $150 \mathrm{ng} / \mathrm{mL}$ per compound except for 12HEPE (600 ng/mL), EPA (3 $\mu \mathrm{g} / \mathrm{mL})$, DHA $(15 \mu \mathrm{g} / \mathrm{mL})$ and ARA $(45 \mu \mathrm{g} / \mathrm{mL}))$ supplemented with different percentages of methanol, 0 to $50 \%, \mathrm{n}=3$ replicates.

Figure 4: Comparative recovery results for the extraction with $60 \mathrm{mg}$ HLB cartridges of spiked plasma samples (final concentration of $150 \mathrm{ng} / \mathrm{mL}$ to every compound except 12HEPE (600 $\mathrm{ng} / \mathrm{mL})$, EPA $(3 \mu \mathrm{g} / \mathrm{mL})$, DHA $(15 \mu \mathrm{g} / \mathrm{mL})$ and ARA $(45 \mu \mathrm{g} / \mathrm{mL}))$ doing

21 the whole process at room temperature and $4{ }^{\circ} \mathrm{C}, \mathrm{n}=3$ replicates.

23 Figure 5: Identification of 11HDoHE using QqQ (a, b) and LIT (c, d) mass spectrometers: a) chromatogram obtained in the MRM mode (QqQ) at the transition of $343>121 \mathrm{~m} / \mathrm{z}$ in plasma sample; b) chromatogram obtained in the MRM mode (QqQ) at the transition of $343>121 \mathrm{~m} / \mathrm{z}$ in standard solution of 11HDoHE; c) corresponding MS/MS spectrum of the identified peak in plasma sample with the LIT; d) corresponding MS/MS spectrum of 11HDoHE standard solution with the LIT. Spectra c and demonstrate the false positive identification of 11HDoHE in plasma by QqQ analysis. 


\section{Table captions}

3 Table 1: Retention times, collision energies and MS/MS transitions for QqQ and LIT

4 spectrometers.

6 Table 2: Linearity (1 to $500 \mathrm{ng} / \mathrm{mL}$ for oxidized derivates and 100 to $50000 \mathrm{ng} / \mathrm{mL}$ for 7 PUFAs), limit of detection (LOD), limit of quantification (LOQ) and repeatability of

8 LC-ESI/MS/MS assay at three concentration levels $(5,100$ and $500 \mathrm{ng} / \mathrm{mL})$ for target

9 compounds for the QqQ and LIT mass spectrometers. "The Repeatability of EPA, ARA and DHA was determined at three different concentration levels: 100, 5000 and 50000 $11 \mathrm{ng} / \mathrm{mL}(\mathrm{n}=5)$.

13 Table 3: Repeatability (RSD) of the global process and recovery results including:

14 Global Recovery (SPE-LC-ESI/MS/MS), SPE Recovery and Matrix Effect (LC15 ESI/MS/MS); for spiked plasma samples $(\mathrm{n}=3)$ to a final concentration of $150 \mathrm{ng} / \mathrm{mL}$ 16 per compound except for 12HEPE (600 ng/mL), EPA $(3 \mu \mathrm{g} / \mathrm{mL})$, DHA $(15 \mu \mathrm{g} / \mathrm{mL})$ and 17 ARA $(45 \mu \mathrm{g} / \mathrm{mL})$. Comparison between QqQ and LIT spectrometers.

Table 4: Average concentration and calculated RSD of studied lipid compounds in ten 20 female rat plasma samples by SPE-LC-LIT. A dilution was needed to calculate 21 17HpDoHE concentration due to his level in samples was higher than the maximum 22 level of calibration.

\section{Electronic Supplementary Material}

25 Figure S1: Cascade of formation to ARA, EPA and DHA oxidized derived compounds 26 from COX, LOX, CYP450 enzyme activities and autoxidation.

28 Figure S2: Individual mass spectrum and a possible explanation of the fragmentation 29 patterns for $\mathrm{PDG}_{3}$ and $\mathrm{PGE}_{3}$ isomers.

31 Figure S3: Optimized SPE methodology for plasma samples containing lipid mediator derivatives. 


\section{Tables}

Table 1

\begin{tabular}{|c|c|c|c|c|c|c|}
\hline \multirow[t]{2}{*}{ Compound } & \multirow[t]{2}{*}{ Retention Time (min) } & \multicolumn{3}{|r|}{ QqQ } & \multicolumn{2}{|r|}{ LIT } \\
\hline & & Collision energy $(\mathrm{eV})$ & Capillary voltage $(\mathrm{eV})$ & MRM quantification ${ }^{q}$ and identification ${ }^{i}$ transitions $(\mathrm{m} / \mathrm{z})$ & Collision energy $(\mathrm{eV})$ & Quantification transition $(\mathrm{m} / \mathrm{z})$ \\
\hline 8iso-PGF PG $_{3 a}$ & 6.79 & $\begin{array}{c}18 \\
14.5\end{array}$ & -68 & $\begin{array}{l}351>307^{q} \\
351>333^{i}\end{array}$ & 30 & $351>253$ \\
\hline $\mathrm{TXB}_{3}$ & 6.97 & $\begin{array}{l}16.0 \\
13.5 \\
\end{array}$ & -52 & $\begin{array}{l}367>169^{q} \\
367>195^{i}\end{array}$ & 19 & $367>195$ \\
\hline $\mathrm{PGD}_{3} / \mathrm{PGE}_{3}$ & 7.67 & $\begin{array}{c}12.5 \\
10\end{array}$ & -40 & $\begin{array}{l}349>269^{q} \\
349>313^{\mathrm{i}}\end{array}$ & 19 & $349>313$ \\
\hline 8iso-PGF $2 a$ & 8.28 & $\begin{array}{l}27.0 \\
24.5 \\
\end{array}$ & -44 & $\begin{array}{l}353>192^{q} \\
353>171^{i}\end{array}$ & 28 & $353>299$ \\
\hline $\mathrm{PGE}_{2}$ & 9.09 & $\begin{array}{l}15.5 \\
10.5 \\
\end{array}$ & -64 & $\begin{array}{l}351>271^{\mathrm{q}} \\
351>315^{\mathrm{i}}\end{array}$ & 20 & $351>315$ \\
\hline $\mathbf{R v D}_{1}$ & 9.80 & $\begin{array}{l}14.0 \\
18.0 \\
\end{array}$ & -56 & $\begin{array}{l}375>141^{\mathrm{q}} \\
375>215^{\mathrm{i}}\end{array}$ & 25 & $375>141$ \\
\hline $\mathbf{P D}_{1}$ & 12.89 & $\begin{array}{l}16.0 \\
19.0\end{array}$ & -64 & $\begin{array}{l}359>153^{q} \\
359>136^{i}\end{array}$ & 30 & $359>153$ \\
\hline $\mathbf{L T B}_{4}$ & 13.79 & $\begin{array}{l}15.0 \\
12.5 \\
\end{array}$ & -80 & $\begin{array}{l}335>195^{\mathrm{q}} \\
335>317^{\mathrm{i}}\end{array}$ & 27 & $335>195$ \\
\hline 15НрЕРE & 17.36 & $\begin{array}{l}9.5 \\
5.0\end{array}$ & -30 & $\begin{array}{l}333>111^{\mathrm{q}} \\
333>315^{\mathrm{i}}\end{array}$ & 20 & $333>315$ \\
\hline 12НpEPE & 17.80 & $\begin{array}{l}5.5 \\
9.0 \\
\end{array}$ & -32 & $\begin{array}{l}333>271^{q} \\
333>151^{i}\end{array}$ & 25 & $333>315$ \\
\hline 15HEPE & 17.92 & $\begin{array}{l}11.0 \\
11.5 \\
\end{array}$ & -56 & $\begin{array}{l}317>219^{q} \\
317>255^{i}\end{array}$ & 27 & $317>219$ \\
\hline 12HEPE & 18.72 & $\begin{array}{l}12.5 \\
12.0\end{array}$ & -56 & $\begin{array}{l}317>179^{q} \\
317>208^{i}\end{array}$ & 27 & $317>179$ \\
\hline 5HEPE & 20.47 & $\begin{array}{l}12.5 \\
10.5\end{array}$ & -56 & $\begin{array}{l}317>115^{\mathrm{q}} \\
317>255^{\mathrm{i}}\end{array}$ & 25 & $317>255$ \\
\hline 17HpDoHE & 21.90 & $\begin{array}{l}9.5 \\
5.5 \\
\end{array}$ & -32 & $\begin{array}{l}359>111^{\mathrm{q}} \\
359>297^{\mathrm{i}}\end{array}$ & 26 & $359>341$ \\
\hline 17HDoHE & 21.94 & $\begin{array}{l}11.5 \\
12.5 \\
\end{array}$ & -64 & $\begin{array}{l}343>281^{q} \\
343>201^{i}\end{array}$ & 27 & $343>245$ \\
\hline 11HETE & 22.09 & $\begin{array}{l}15.0 \\
12.5 \\
\end{array}$ & -80 & $\begin{array}{l}319>167^{q} \\
319>301^{i}\end{array}$ & 30 & $319>167$ \\
\hline 12HETEd $_{8}$ & 22.66 & $\begin{array}{l}13.5 \\
12.0 \\
\end{array}$ & -68 & $\begin{array}{l}325>182^{\mathrm{q}} \\
325>307^{\mathrm{i}}\end{array}$ & 30 & $325>307$ \\
\hline 11HDoHE & 23.20 & $\begin{array}{l}14.0 \\
12.5 \\
\end{array}$ & -60 & $\begin{array}{l}343>121^{\mathrm{q}} \\
343>149^{\mathrm{i}}\end{array}$ & 27 & $343>149$ \\
\hline 4HDoHE & 23.64 & $\begin{array}{l}13.5 \\
10.5\end{array}$ & -64 & $\begin{array}{l}343>101^{\mathrm{q}} \\
343>281^{\mathrm{i}}\end{array}$ & 27 & $343>281$ \\
\hline EPA & 24.43 & $\begin{array}{l}10.0 \\
12.5\end{array}$ & -64 & $\begin{array}{l}301>257^{q} \\
301>203^{i}\end{array}$ & 27 & $301>257$ \\
\hline DHA & 24.93 & $\begin{array}{c}9.5 \\
11.5 \\
\end{array}$ & -38 & $\begin{array}{l}327>283^{q} \\
327>229^{i}\end{array}$ & 30 & $327>283$ \\
\hline$\overline{\text { ARA }}$ & 25.10 & $\begin{array}{l}13.5 \\
12.0\end{array}$ & -76 & $\begin{array}{l}303>205^{q} \\
303>259^{i}\end{array}$ & 30 & $303>259$ \\
\hline
\end{tabular}


Table 2

\begin{tabular}{|c|c|c|c|c|c|c|c|c|c|c|c|c|}
\hline \multirow[t]{3}{*}{ Compound } & \multicolumn{2}{|c|}{$\overline{\mathbf{R}^{2}}$} & \multicolumn{2}{|c|}{ LOD(ng/mL) } & \multicolumn{2}{|c|}{ LOQ(ng/mL) } & \multicolumn{6}{|c|}{ Repeatability (RSD \% n=5) } \\
\hline & \multirow[t]{2}{*}{ QqQ } & \multirow[t]{2}{*}{ LIT } & \multirow[t]{2}{*}{ QqQ } & \multirow[t]{2}{*}{ LIT } & \multirow[t]{2}{*}{ QqQ } & \multirow[t]{2}{*}{ LIT } & \multicolumn{3}{|c|}{ QqQ } & \multicolumn{3}{|c|}{ LIT } \\
\hline & & & & & & & 5ng/mL & 100ng/mL & $500 \mathrm{ng} / \mathrm{mL}$ & $5 \mathrm{ng} / \mathrm{mL}$ & 100ng/mL & $500 \mathrm{ng} / \mathrm{mL}$ \\
\hline 12НрЕРЕ & 0.9868 & 0.9991 & 2 & 3 & 6 & 10 & 22 & 4 & 5 & 3 & 3 & 2 \\
\hline 15НрЕРЕ & 0.9868 & 0.9991 & 0.4 & 0.6 & 1.5 & 2 & 16 & 4 & 2 & 3 & 3 & 2 \\
\hline 17HpDoHE & 0.9879 & 0.9903 & 1 & 1 & 3.5 & 4 & 11 & 7 & 1 & 5 & 3 & 7 \\
\hline 11HDoHE & 0.9965 & 0.9957 & 0.9 & 0.1 & 3 & 0.3 & 7 & 4 & 4 & 3 & 1 & 2 \\
\hline 11HETE & 0.9997 & 0.9931 & 0.1 & 0.02 & 0.4 & 0.07 & 8 & 2 & 1 & 4 & 9 & 5 \\
\hline 12HEPE & 0.9974 & 0.9939 & 0.7 & 0.08 & 2 & 0.3 & 7 & 6 & 2 & 4 & 1 & 1 \\
\hline 12HETEd8 & & & 0.003 & 0.002 & 0.01 & 0.006 & 3 & 3 & 4 & 5 & 6 & 4 \\
\hline 15HEPE & 0.9997 & 0.9968 & 0.5 & 0.07 & 1.5 & 0.2 & 10 & 3 & 2 & 4 & 1 & 1 \\
\hline 17HDoHE & 0.9999 & 0.9947 & 0.5 & 0.2 & 2 & 0.7 & 12 & 3 & 6 & 6 & 1 & 1 \\
\hline 4HDoHE & 0.9921 & 0.9922 & 1.5 & 0.3 & 5 & 1 & 5 & 1 & 2 & 7 & 5 & 4 \\
\hline 5HEPE & 0.9960 & 0.9938 & 0.5 & 0.1 & 2 & 0.3 & 4 & 3 & 6 & 4 & 1 & 1 \\
\hline LTB4 & 0.9994 & 0.9954 & 0.3 & 0.03 & 1 & 0.1 & 6 & 1 & 1 & 3 & 2 & 1 \\
\hline PD1 & 0.9997 & 0.9971 & 0.3 & 0.02 & 1 & 0.1 & 6 & 2 & 0.05 & 5 & 1 & 2 \\
\hline PGD3/PGE3 & 0.9992 & 0.9985 & 0.2 & 0.1 & 1 & 0.3 & 2 & 1 & 3 & 1 & 1 & 2 \\
\hline PGE2 & 0.9993 & 0.9925 & 0.1 & 0.05 & 0.4 & 0.2 & 6 & 1 & 1 & 2 & 1 & 1 \\
\hline RvD1 & 0.9995 & 0.9958 & 0.1 & 0.02 & 0.4 & 0.07 & 6 & 1 & 2 & 1 & 1 & 2 \\
\hline TXB3 & 0.9970 & 0.9971 & 0.4 & 0.1 & 1 & 0.5 & 5 & 2 & 1 & 4 & 1 & 1 \\
\hline PGF2 $\alpha$ & 0.9992 & 0.9936 & 1 & 0.06 & 3 & 0.2 & 4 & 4 & 3 & 8 & 3 & 2 \\
\hline PGF3a & 0.9997 & 0.9959 & 7 & 0.4 & 23 & 1.5 & 5 & 3 & 2 & 2 & 1 & 1 \\
\hline ARA & 0.9997 & 0.9934 & 0.1 & 0.04 & 0.3 & 0.1 & 6 & 3 & *1 & 3 & 5 & 9 \\
\hline EPA & 0.9978 & 0.9959 & 0.1 & 0.01 & 0.2 & 0.04 & 6 & 3 & 2 & 3 & 4 & 7 \\
\hline DHA & 0.9876 & 0.9860 & 0.1 & 0.01 & 0.2 & 0.04 & 2 & 2 & 3 & 3 & 5 & 8 \\
\hline
\end{tabular}


$1 \quad$ Table 3

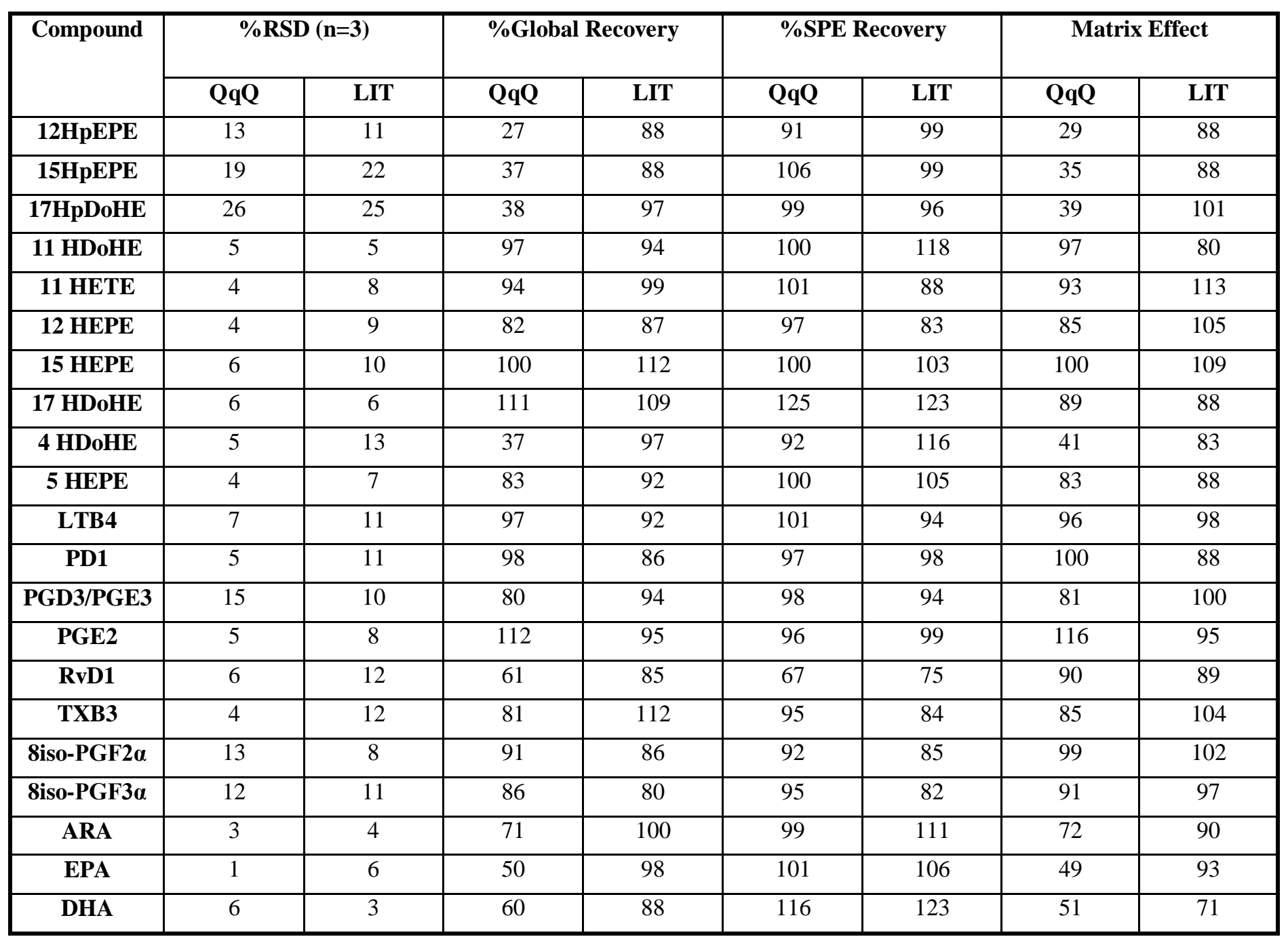

2 
$1 \quad$ Table 4

2

\begin{tabular}{|c|c|}
\hline Compound & $\begin{array}{c}\text { Average sample } \\
\text { concentration }(\mathbf{n g} / \mathbf{m L}) \\
(\mathbf{n}=\mathbf{1 0})\end{array}$ \\
\hline 17HpDoHE & $2183 \pm 240$ \\
\hline 11 HETE & $3 \pm 0.5$ \\
\hline 12 HEPE & $249 \pm 69$ \\
\hline PGE2 & $10 \pm 1$ \\
\hline TXB3 & $3 \pm 0.4$ \\
\hline ARA & $59446 \pm 10105$ \\
\hline EPA & $8370 \pm 1255$ \\
\hline DHA & $48632 \pm 7781$ \\
\hline
\end{tabular}




\section{Figures}

\section{Figure 1}
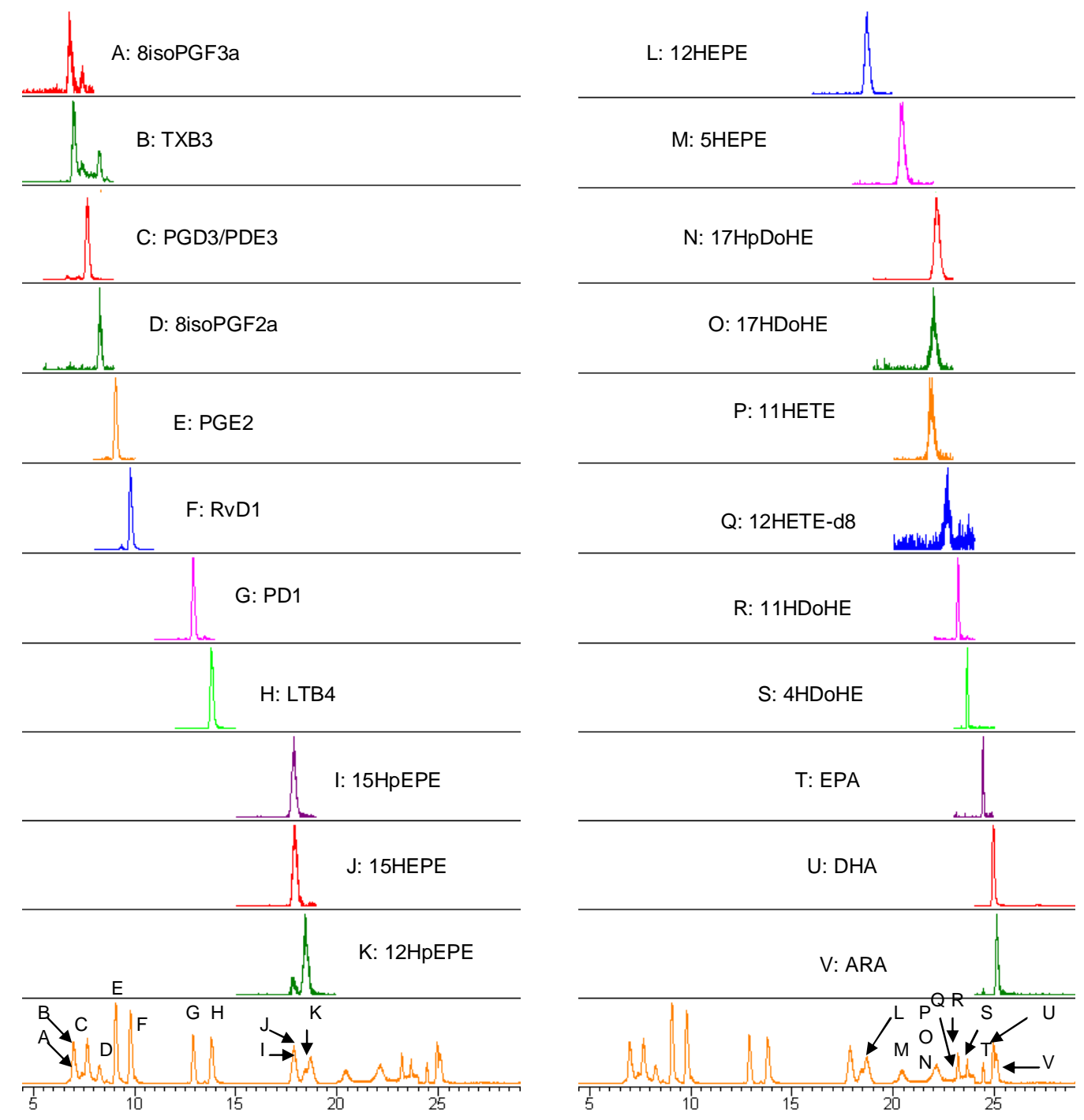
$1 \quad$ Figure 2

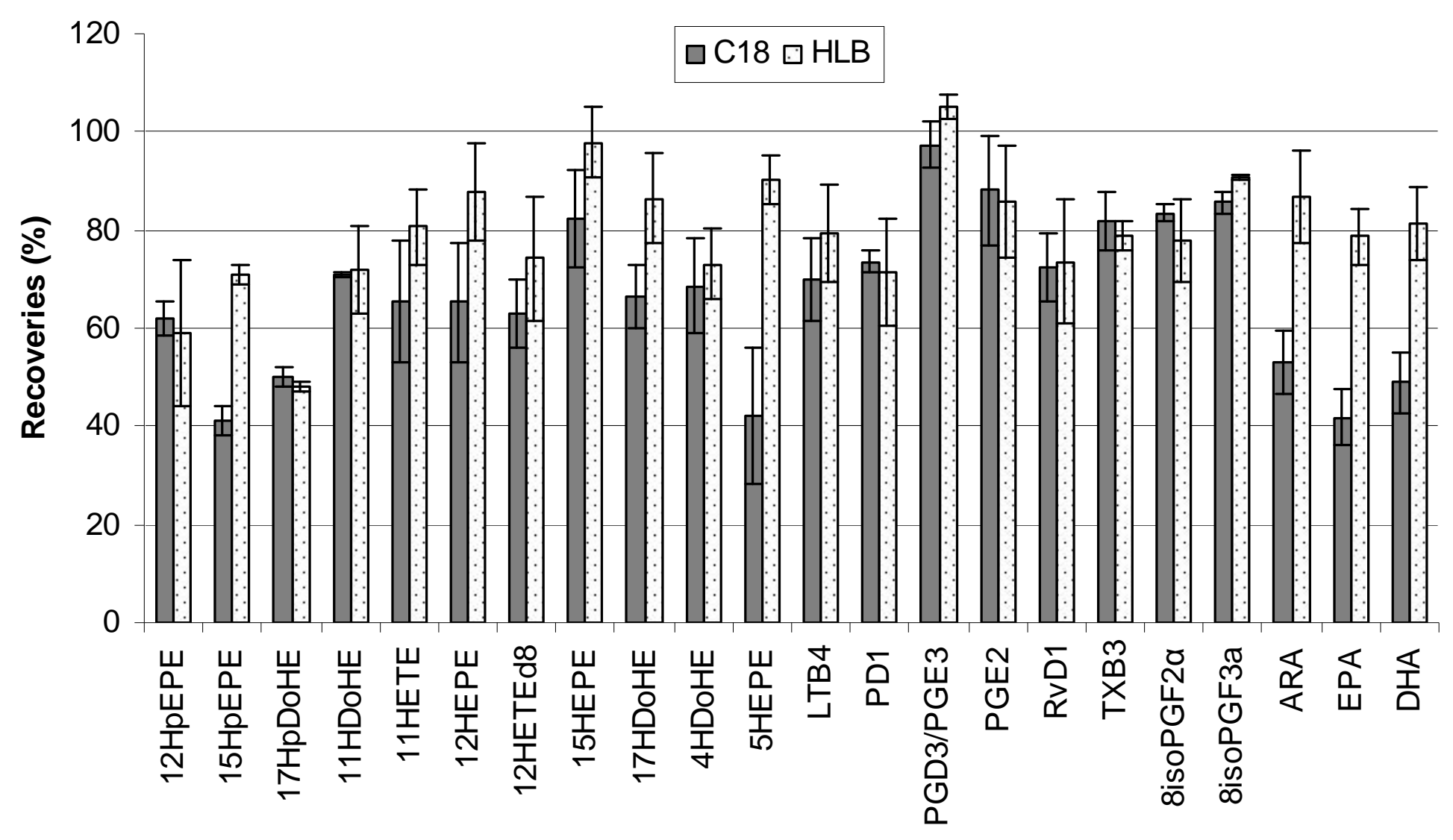


Figure 3

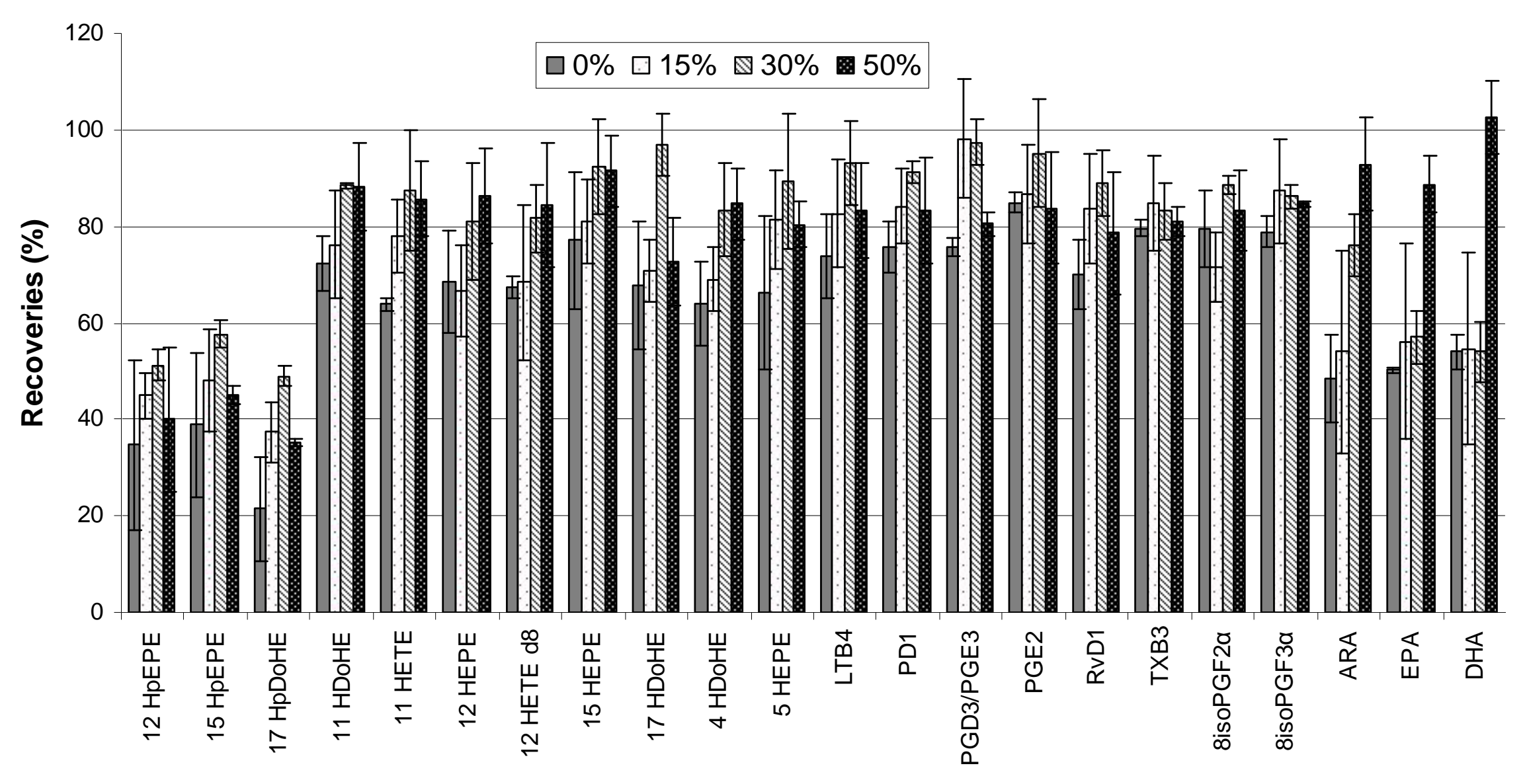




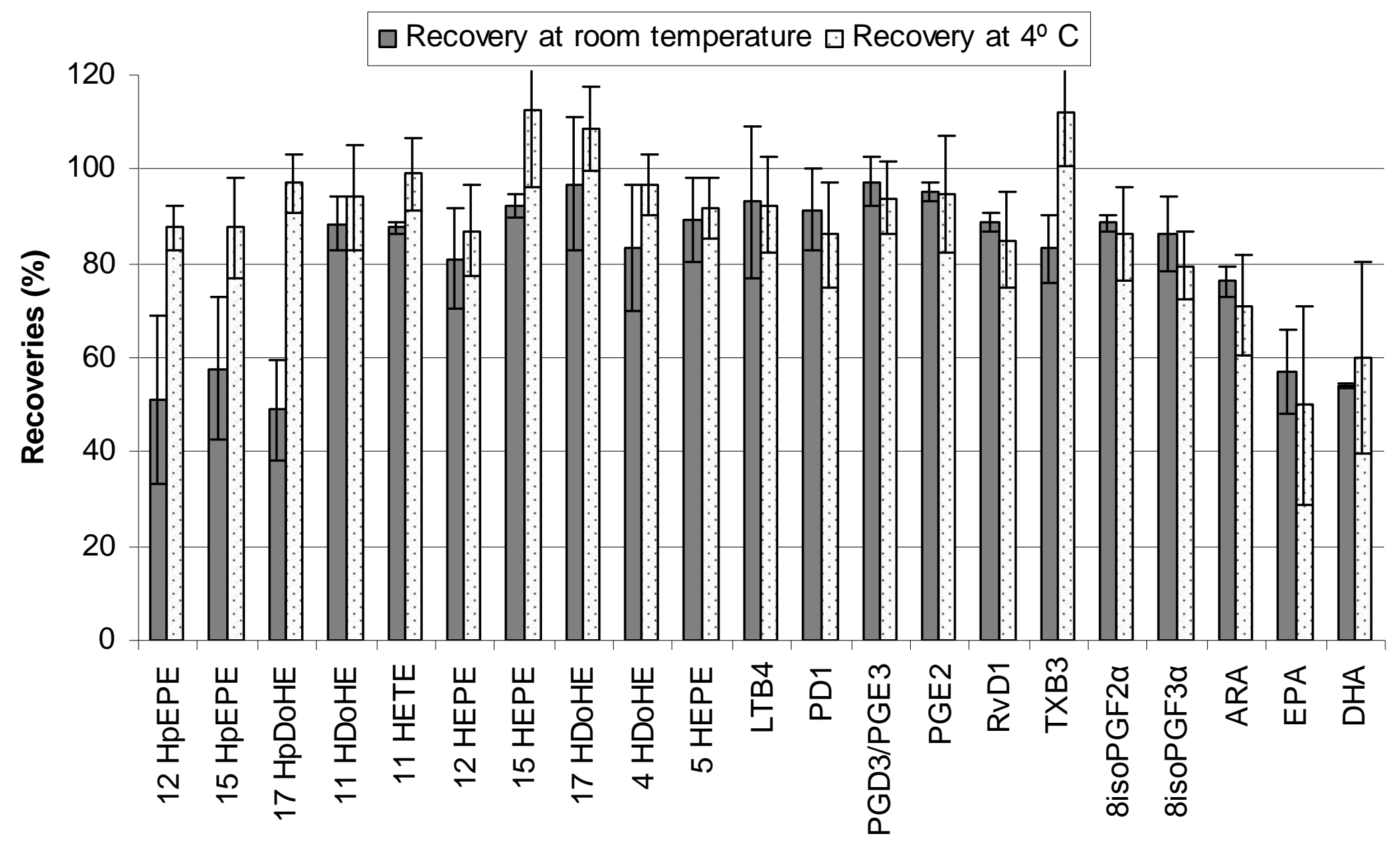




\section{Figure 5}
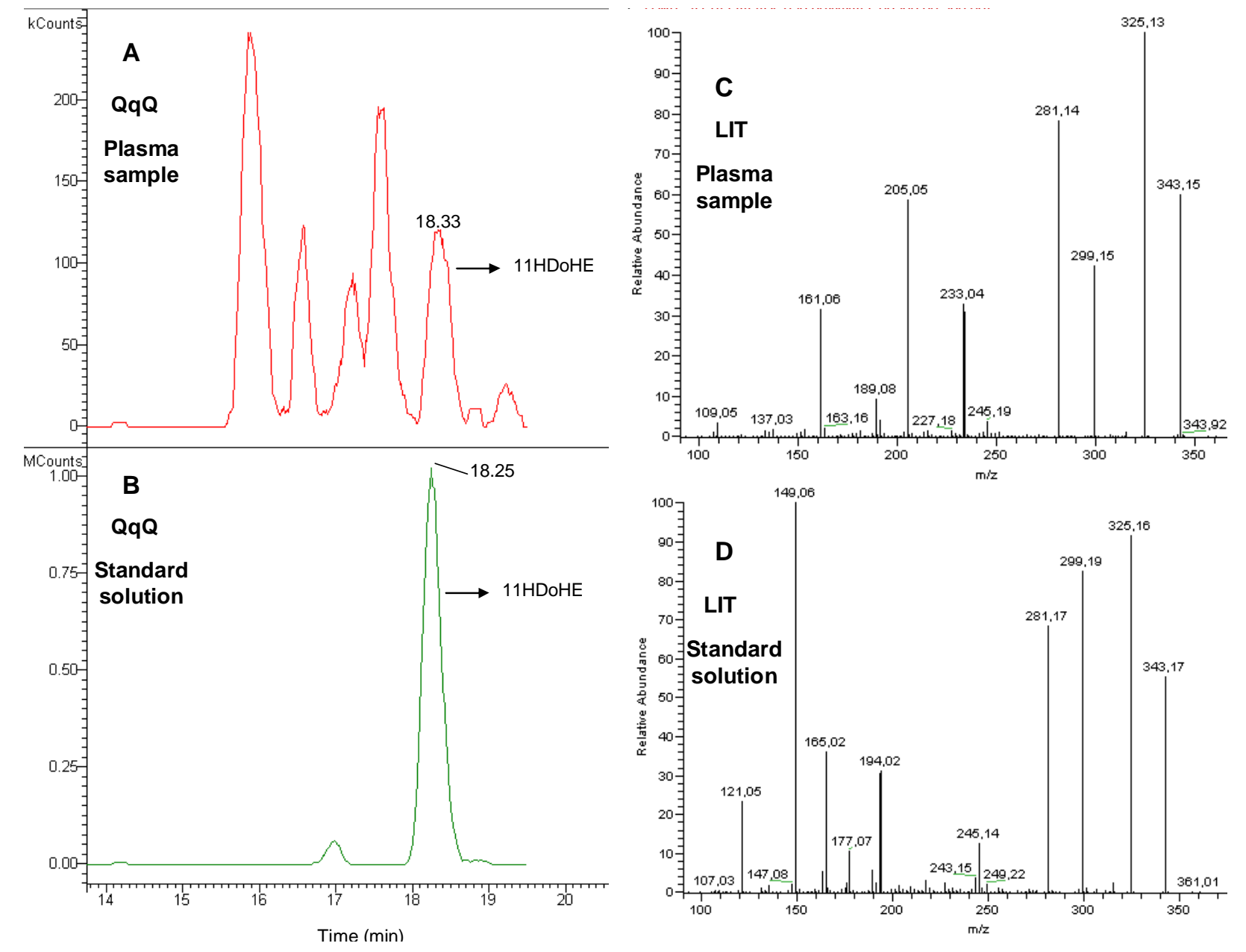


\section{Electronic Supplementary material}

Figure S1
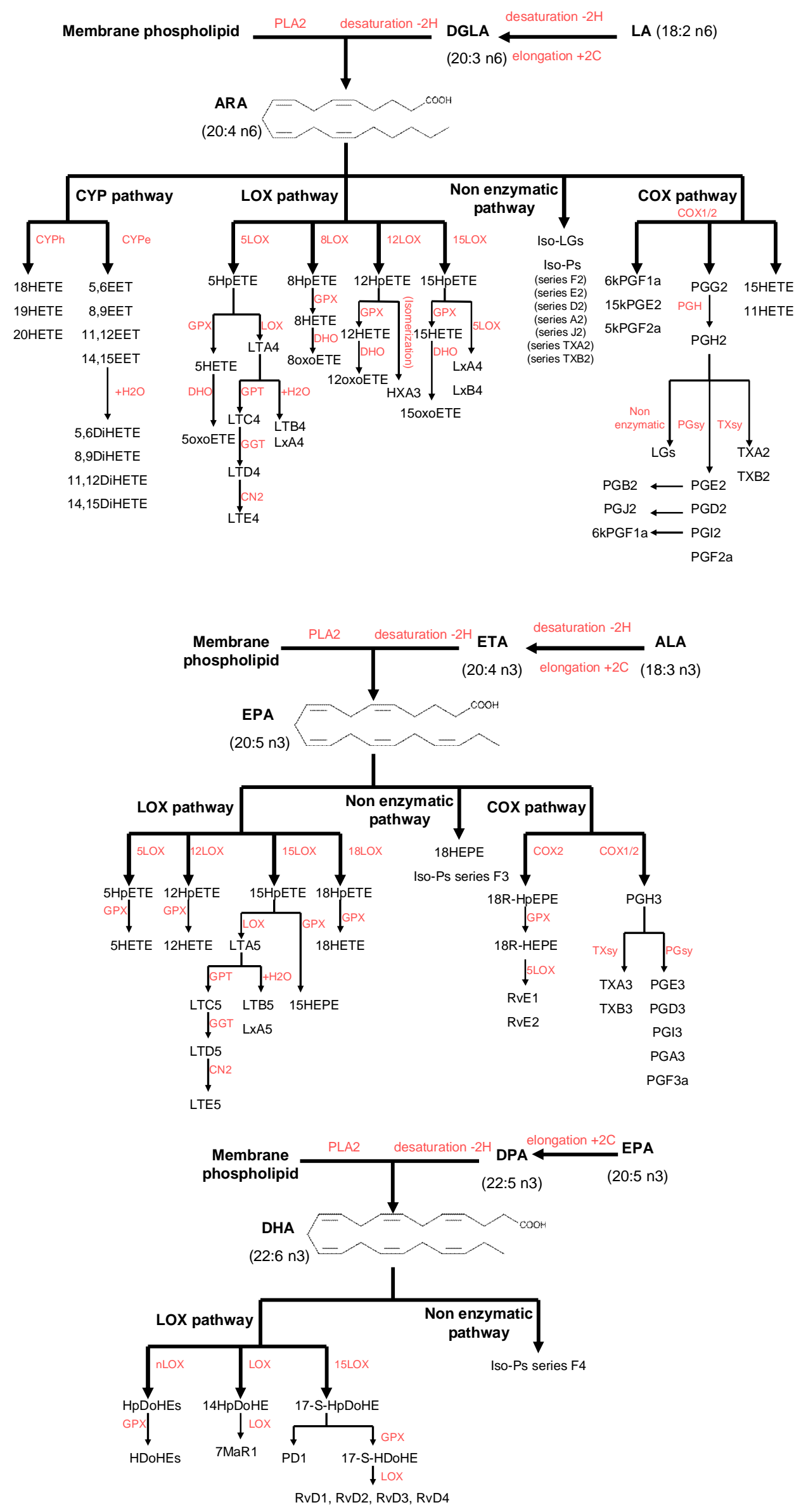


\section{$1 \quad$ Figure S2}

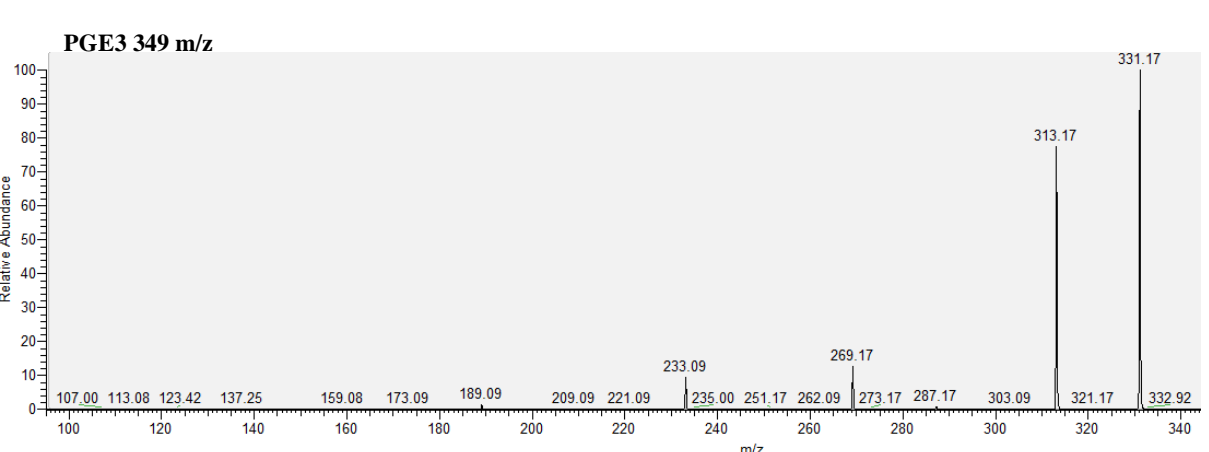

PGD3 349 m/z

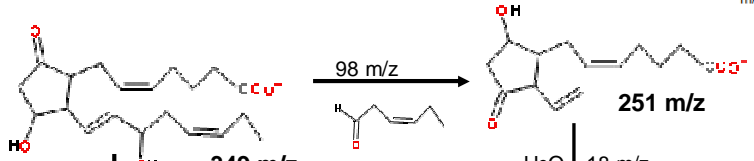

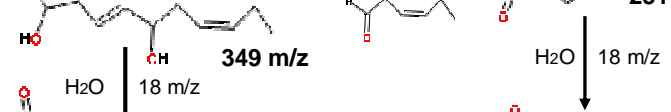

(18)
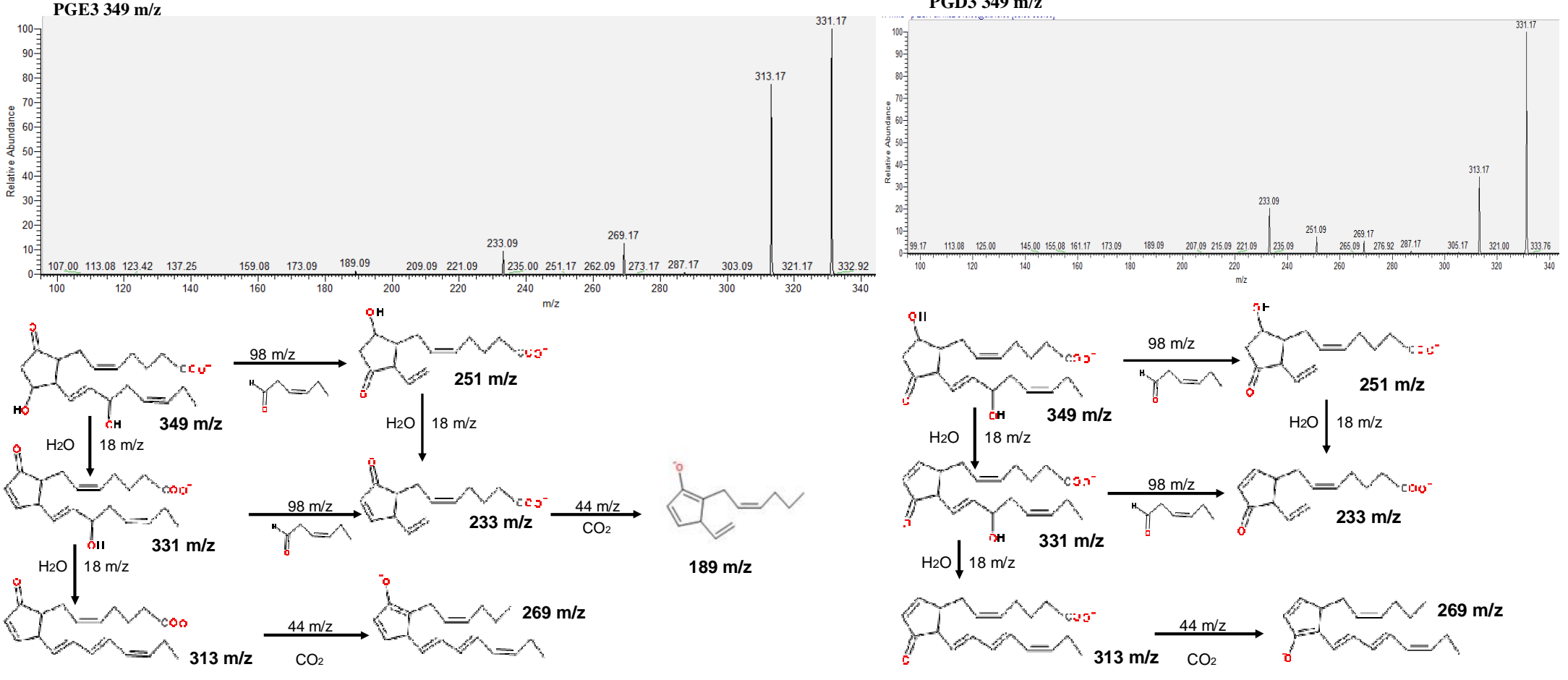

$189 \mathrm{~m} / \mathrm{z}$

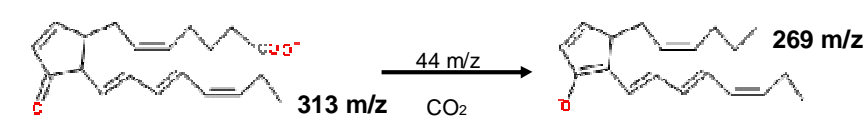




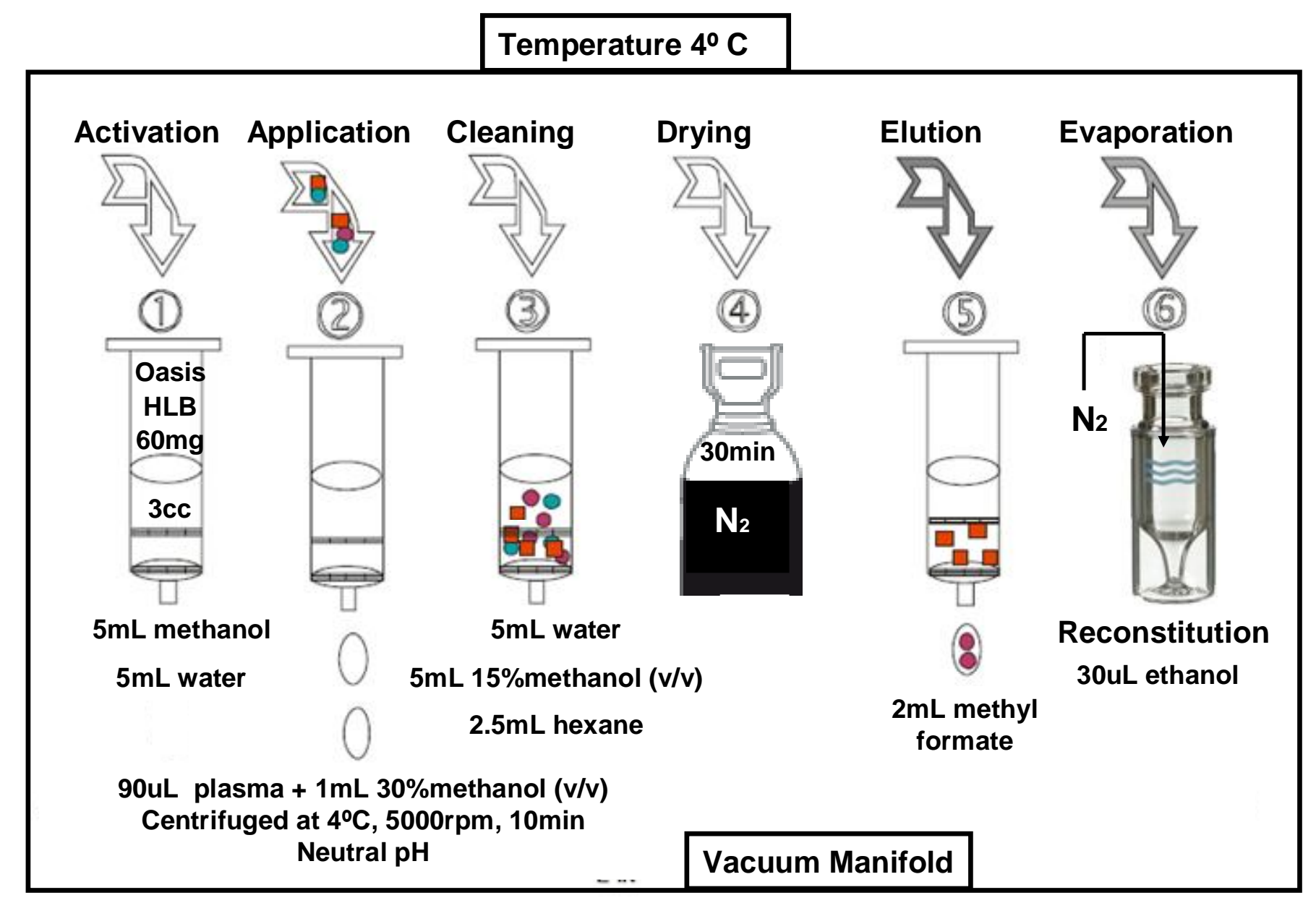

